\title{
Detecting fibered strongly quasi-positive links
}

\author{
Alberto Cavallo \\ Max Planck Institute for Mathematics, \\ Bonn 53111, Germany \\ cavallo@mpim-bonn.mpg.de
}

\begin{abstract}
We prove that an $n$-component fibered link $L$ in $S^{3}$ is strongly quasi-positive if and only if $\tau(L)=g_{3}(L)+n-1$, where $g_{3}(L)$ denotes the Seifert genus and $\tau$ is the Ozsváth-Szabó concordance invariant. We also provide a table which contains a list of some fibered prime links with at most 9 crossings; and we explicitly determine the ones that are strongly quasi-positive and their maximal self-linking number.
\end{abstract}

\section{Introduction}

It is a very known result in low dimensional topology that every contact 3-manifold can be presented by an open book decomposition; this is a pair $(L, \pi)$ where $L$ is a smooth link in $M$ and $\pi: M \backslash L \rightarrow S^{1}$ is a locally trivial fibration whose fiber's closure is a compact surface $F$ with $\partial F=L$. Such an $L$ is then called a fibered link in $M$ and $F$ is a fibered surface for $L$.

In this paper we are only interested in the case where the ambient manifold $M$ is the 3-sphere. Fibered links in $S^{3}$ possess special properties; in fact, those links have unique fibered surfaces up to isotopy and, once such a surface is fixed, the fibration $\pi$ is also completely determined. It follows that in $S^{3}$ we can define fibered links as the links whose complement fibers over the circle.

Since an open open book decomposition $(L, \pi)$ always determines a contact structure, we obtain a partition of fibered links in $S^{3}$ by saying that $L$ carries the structure $\xi_{L}$. In particular, it is a consequence of classical results of Harer and Stallings [9, 22, and the Giroux correspondence [8], that the subset of fibered links, inducing the unique tight structure $\xi_{\text {st }}$ on $S^{3}$, coincides with the set of strongly quasipositive links. We recall that a link is said strongly quasi-positive if it can be written as closure of the composition of $d$-braids of the form

$$
\left(\sigma_{i} \cdots \sigma_{j-2}\right) \sigma_{j-1}\left(\sigma_{i} \cdots \sigma_{j-2}\right)^{-1} \quad \text { for some } \quad d \geqslant j \geqslant i+2 \geqslant 3
$$

or

$$
\sigma_{i} \quad \text { for } \quad i=1, \ldots, d-1,
$$

where $\sigma_{1}, \ldots, \sigma_{d-1}$ are the Artin generators of the $d$-braids group.

Remark 1.1. Fibered and strongly quasi-positive links in $S^{3}$ are connected transverse $\mathbb{C}$-links in the sense of [3].

The main result of our paper is a criterion for detecting exactly which fibered links in $S^{3}$ are strongly quasi-positive, and then carry the contact structure $\xi_{\text {st }}$, generalizing results of Hedden [11] (for knots) and Boileau, Boyer and Gordon [1] (for non-split alternating links). We recall that Ozsváth, Stipsicz 
and Szabó defined the $\tau$-set (of $2^{n-1}$ integers) of an $n$-component link in [17, using the link Floer homology group $\mathrm{cHFL}^{-}$, and they denote with $\tau_{\max }$ and $\tau_{\min }$ its maximum and minimum. Moreover, the $\tau$-set contains $\tau$, the concordance invariant defined in [2] by the author.

Theorem 1.2. A fibered link $L$ in $S^{3}$ is strongly quasi-positive if and only if $\tau(L)=g_{3}(L)+n-1$ and, in this case, one also has $\tau(L)=\tau_{\max }(L)$.

Combining Theorem 1.2 with [6, Theorem 1.1] and [3, Theorem 1.4] we easily obtain the following corollary.

Corollary 1.3. For a fibered link $L$ the fact that $\tau(L)=g_{3}(L)+n-1$ immediately implies $S L(L)=$ $2 \tau(L)-n$, where $S L(L)$ denotes the maximal self-linking number of $L$.

Furthermore, we give a table where we list all prime alternating fibered links with crossing number at most 9, together with the prime non-alternating fibered ones with crossing number at most 7 , and we denote whether they are strongly quasi-positive. We plan to expand the table in the future, but unfortunately at the moment we do not have knowledge of any program to compute $\tau$ for links.

The paper is organized as follows: in Section 2 we recall the basics properties of link Floer homology and fibered surfaces in the 3-sphere; moreover, we prove Theorem 1.2, Finally, in Section 3 we present our table of small prime fibered links and we explain how to read it.

Funding: The author has a post-doctoral fellowship at the Max Planck Institute for Mathematics in Bonn.

\section{Proof of the main theorem}

\subsection{Link Floer homology}

In [21] Ozsváth and Szabó describe how to construct the chain complex $\left(\widehat{C F L}_{*}(\mathcal{D}), \widehat{\partial}\right)$ from a Heegaard diagram $\mathcal{D}=(\Sigma, \alpha, \beta, \mathbf{w}, \mathbf{z})$, where the sets of basepoints both contain $n$ elements. They show that, under some conditions, the diagram $\mathcal{D}$ represents an $n$-component link $L$.

If we ignore the information given by $\mathbf{z}$ then $\mathcal{D}$ is just a (multi-pointed) Heegaard diagram for $S^{3}$ and, using the same procedure, we obtain the complex $\left(\widehat{C F}_{*}(\mathcal{D}), \widehat{\partial}\right)$, see [18]. When $n=1$ the homology of the latter complex is denoted by $\widehat{H F}_{*}\left(S^{3}\right) \cong \mathbb{F}_{0}$, where $\mathbb{F}$ is the field with two elements. In general, we have that

$$
H_{*}(\widehat{C F}(\mathcal{D})) \cong \widehat{H F}_{*}\left(S^{3}\right) \otimes\left(\mathbb{F}_{-1} \oplus \mathbb{F}_{0}\right)^{\otimes n-1}
$$

hence, if $\mathcal{D}_{1}$ and $\mathcal{D}_{2}$ have the same number of basepoints then $\widehat{C F}_{*}\left(\mathcal{D}_{1}\right)$ is chain homotopy equivalent to $\widehat{C F}_{*}\left(\mathcal{D}_{2}\right)$.

The homology of $\widehat{C F L}_{*}(\mathcal{D})$, following the notation of the author in [2], is denoted by $\widehat{\mathcal{H F}}_{*}(L)$, but for what we said before this group is graded isomorphic to $H_{*}(\widehat{C F}(\mathcal{D}))$. The difference, in the case of links, is given by the fact that the basepoints in $\mathbf{z}$ define the Alexander (collapsed) increasing filtration $\mathcal{A}^{s} \widehat{C F L}_{*}(\mathcal{D})$ with $s \in \mathbb{Z}$. Such a filtration descends into homology in the following way: consider the quotient projection $\pi_{d}: \operatorname{Ker} \widehat{\partial}_{d} \rightarrow \widehat{\mathcal{H F}}_{d}(L)$, where $\widehat{\partial}_{d}: \widehat{C F L}_{d}(\mathcal{D}) \rightarrow \widehat{C F L}_{d-1}(\mathcal{D})$ is the restriciton of $\widehat{\partial}$ to $\widehat{C F L}_{d}(\mathcal{D})$, and define

$$
\operatorname{Ker} \widehat{\partial}_{d, s}=\operatorname{Ker} \widehat{\partial}_{d} \cap \mathcal{A}^{s} \widehat{C F L}_{d}(\mathcal{D}) \text {. }
$$

Then we say that

$$
\mathcal{A}^{s} \widehat{\mathcal{H F}}_{d}(L)=\pi_{d}\left(\operatorname{Ker} \widehat{\partial}_{d, s}\right) \subset \widehat{\mathcal{H F}}_{d}(L)
$$


for any $d \in \mathbb{Z}$. It is important to observe that $\mathcal{A}^{s} \widehat{\mathcal{H F}}_{*}(L)$ is not the same homology group obtained by just taking the homology of the Alexander level $s$ of $\widehat{C F L}(\mathcal{D})$, which is instead denoted with $H_{*}\left(\mathcal{A}^{s} \widehat{C F L}(\mathcal{D})\right)$; more specifically, the latter group is obtained by first restricting $\widehat{C F L}_{d}(\mathcal{D})$ and $\widehat{\partial}_{d}$ to the Alexander level $s$ and then extracting the homology. In particular, note that $\mathcal{A}^{s} \widehat{\mathcal{H F}}_{*}(L)$ is, by definition, always a subgroup of $\widehat{\mathcal{H F} \mathcal{L}_{*}}(L)$, while this needs not be true for $H_{*}\left(\mathcal{A}^{s} \widehat{C F L}(\mathcal{D})\right)$.

In addition, we write $\widehat{H F L}_{*, *}(L)$ for the homology of the graded object associated to $\mathcal{A} \widehat{C F L}_{*}(\mathcal{D})$, which is the bigraded complex $\left(\operatorname{gr}_{*, *}(\mathcal{D}), \operatorname{gr}(\widehat{\partial})\right)$ defined as follows, see [17]: we have that $\operatorname{gr}_{d, s}(\mathcal{D})$ is the subspace of $\mathcal{A}^{s} \widehat{C F L}_{d}(\mathcal{D})$ spanned by generators that are not in $\mathcal{A}^{s-1} \widehat{C F L}_{d}(\mathcal{D})$, while $\operatorname{gr}(\widehat{\partial})$ is the component of $\widehat{\partial}$ which preserves the Alexander grading, so that $\operatorname{gr}_{d, s}(\widehat{\partial}):=\left.\operatorname{gr}(\widehat{\partial})\right|_{\operatorname{gr}_{d, s}(\mathcal{D})}: \operatorname{gr}_{d, s}(\mathcal{D}) \rightarrow$ $\operatorname{gr}_{d-1, s}(\mathcal{D})$.

We conclude this subsection by recalling that in [2, Theorem 1.3] the invariant $\tau_{\min }(L)$ was shown by the author to coincide with the minimal integer $s$ such that $\mathcal{A}^{s} \widehat{\mathcal{H F}}_{*}(L)$ is non-zero.

\subsection{Fibered surfaces and the Thurston norm}

It is a result of Ghiggini [7] and Ni [15] that link Floer homology detects fibered links in the 3-sphere.

Theorem 2.1 (Ghiggini-Ni). A link $L \hookrightarrow S^{3}$ with $n$-components is fibered if and only if $\operatorname{dim} \widehat{H F L}_{*, \text { stop }}(L)=1$, where $s_{\text {top }}$ is the maximal $s \in \mathbb{Z}$ such that the group $\widehat{H F L}_{*, s}(L)$ is non-zero.

We recall that $\|L\|_{T}$ denotes the evaluation of the Thurston semi-norm [23] at the homology class represented by the Seifert surfaces of $L$; and it is defined as

$$
\|L\|_{T}=o(L)-\max \{\chi(\Sigma)\}
$$

where $\Sigma$ is a compact and oriented surface in $S^{3}$, such that $\partial \Sigma=L$, and $o(L)$ is the number of unknotted unknots in $L$.

Hence, it follows from [16] that, for an $L$ as in Theorem 2.1, one has $2 s_{\text {top }}=\|L\|_{T}+n$, assuming that $L$ is not the unknot. Morever, we have the following result.

Proposition 2.2. If $L$ is a fibered $n$-component link in $S^{3}$ then $s_{\text {top }}=g_{3}(L)+n-1$.

Proof. The statement is clearly true for the unknot and it is a classical result, see [13, Chapters 4 and $5]$, that fibered surfaces are always connected and minimize the Thurston norm of $L$. Therefore, we have

$$
s_{\text {top }}=\frac{-\chi(F)+n}{2}=\frac{-(2-2 g(F)-n)+n}{2}=g(F)+n-1,
$$

where $F$ is a fibered surface for $L$.

Now, if $L$ admits a Seifert surface $T$, with $g(T)<g(F)$, then this contradicts the fact that $F$ minimizes $\|L\|_{T}$ and the claim follows.

\subsection{The contact invariant $c(L)$}

In order to prove Theorem 1.2, we need to recall the original definition of the contact invariant $\widehat{c}(\xi) \in \widehat{H F}\left(S^{3}\right)$ from [19] and adapt it to our setting. In [19] Ozsváth and Szabó, for a given fibered knot $K \hookrightarrow S^{3}$, constructed a Heegaard diagram $\mathcal{D}_{K}$, representing the mirror image of the knot $K$, with a distinguished intersection point $c(K)$ such that

$$
A(c(K))=-g_{3}(K) \quad \text { and } \quad A(y)>-g_{3}(K)
$$


for every other intersection point $y$ in $\mathcal{D}_{K}$. In particular, this gives that

$$
H_{*}\left(\mathcal{A}^{-g_{3}(K)} \widehat{C F K}\left(\mathcal{D}_{K}\right)\right) \cong \mathbb{F}
$$

and

$$
H_{*}\left(\mathcal{A}^{s} \widehat{C F K}\left(\mathcal{D}_{K}\right)\right) \cong\{0\} \quad \text { for any } s<-g_{3}(K) .
$$

This construction works exactly in the same way for an $n$-component fibered link $L$ : only that now one has $A(c(L))=-g$ with $g \in \mathbb{Z}$ and $c(L)$ is still the unique minimal Alexander grading intersection point in $\mathcal{D}_{L}$.

Lemma 2.3. Suppose that $L, c(L)$ and $g$ are as before. Then we have that $g=g_{3}(L)+n-1$.

Proof. The element $c(L)$ is necessarily a cycle and $[c(L)]$ has to be non-zero in $\widehat{H F L}\left(L^{*}\right)$; hence, we have that $A(c(L))$ is the minimal integer $s$ such that $\widehat{H F L}_{*, s}\left(L^{*}\right)$ is non-zero. Because of the symmetries of $\widehat{H F L}(L)$, see [20], and Proposition 2.2 this means that $A(c(L))=-g_{3}(L)-n+1=-g$.

If we consider the inclusion $i^{L,-g}: \mathcal{A}^{-g} \widehat{C F L}\left(\mathcal{D}_{L}\right) \hookrightarrow \widehat{C F}\left(\mathcal{D}_{L}\right)$ then we can see $c(L)$ as a cycle in $\widehat{C F}\left(\mathcal{D}_{L}\right)$ by dropping the information about the Alexander grading.

The element $c(L)$ can then be identified with the contact element defined in [4], using multi-pointed Legendrian Heegaard diagrams, generalizing the presentation of Honda, Kazez and Matić, see [12]. This means that

$$
[c(L)]=\widehat{c}\left(\xi_{L}\right) \otimes \mathbf{e}_{1-n} \in \widehat{H F}\left(S^{3}\right) \otimes\left(\mathbb{F}_{-1} \oplus \mathbb{F}_{0}\right)^{\otimes n-1},
$$

where $\mathbf{e}_{1-n}$ is the unique generator of $\left(\mathbb{F}_{-1} \oplus \mathbb{F}_{0}\right)^{\otimes n-1}$ in grading $1-n$. In particular, we can state the following lemma.

Lemma 2.4. Suppose that $L$ is a fibered link and $\xi_{L}$ is the contact structure on $S^{3}$ carried by $L$. Then $c(L)$ is zero in homology whenever $\xi_{L}$ is overtwisted.

Proof. From [19] one has $\widehat{c}\left(\xi_{L}\right)=[0]$ when $\xi_{L}$ is overtwisted. Hence, the homology class $[c(L)]$ is equal to zero for Equation (2.1).

From the definition of $c(L)$ we observe that $c(L)$ is non-zero in homology if and only if $-g$ is the minimal $t$ such that the map

$$
i_{*}^{L, t}: H_{*}\left(\mathcal{A}^{t} \widehat{C F L}\left(\mathcal{D}_{L}\right)\right) \longrightarrow \widehat{\mathcal{H F} \mathcal{L}_{*}}\left(L^{*}\right) \cong \widehat{H F}\left(S^{3}\right) \otimes\left(\mathbb{F}_{-1} \oplus \mathbb{F}_{0}\right)^{\otimes n-1}
$$

induced by the inclusion $i^{L, t}$ is non-trivial.

Proof of Theorem 1.2. If $L$ is strongly quasi-positive then from [3, Theorem 1.4] one has $\tau(L)=$ $\tau_{\max }(L)=g_{3}(L)+n-1$. Suppose now that $\tau_{\max }(L)=g_{3}(L)+n-1=g$, see Lemma 2.3, we want to prove that $L$ is strongly quasi-positive.

We have that

$$
-g=-\tau_{\max }(L)=\tau_{\min }\left(L^{*}\right)=\min _{s \in \mathbb{Z}}\left\{\mathcal{A}^{s} \widehat{\mathcal{H F} \mathcal{L}}\left(L^{*}\right) \text { is non-zero }\right\}=\min _{s \in \mathbb{Z}}\left\{i_{*}^{L, s} \text { is non-trivial }\right\}
$$

from the definition of $\tau$-set given in [2]. Since $L$ is fibered, the combination of Equations (2.2) and (2.3) tells us precisely that $c(L)$ is non-zero in homology and, applying Lemma 2.4 that $\xi_{L}=\xi_{\mathrm{st}}$.

At this point, we conclude by recalling that a fibered link $L$ carries the tight structure on $S^{3}$ if and only if is strongly quasi-positive, as we observed in the introduction. 


\section{Table of small prime fibered strongly quasi-positive links}

Using Theorem 1.2 and some computation we can detect exactly which prime links are fibered and, among these, which ones are strongly quasi-positive. Those data are enclosed in Table 1. We denote the links following the notation on Linkinfo database [14. Therefore, the number inside brackets identifies the relative orientation of the link. Clearly, being fibered and strongly quasi-positive does depend on relative orientations. Moreover, a link $L$ is fibered if and only if $L^{*}$ is fibered; while when in Table 1 we write that $L$ is strongly quasi-positive, we mean that at least one between $L$ and $L^{*}$ is. In fact, the following result holds.

Theorem 3.1. Suppose that $L$ is a strongly quasi-positive link in $S^{3}$. Then its mirror image $L^{*}$ is also strongly quasi-positive if and only if $L$ is an unlink.

Theorem 3.1 follows from a more general result of Hayden [10, but we can prove it directly using only link Floer homology. Let us start from the following lemma.

Lemma 3.2. If $L$ is strongly quasi-positive then the same its true for $L^{\prime}$, where $L=L^{\prime} \sqcup \bigcirc_{o}(L)$ and $O_{\ell}$ is the $\ell$-unlink. Furthermore, one has $\|L\|_{T}=\left\|L^{\prime}\right\|_{T}$.

Proof. The link $L$ bounds a quasi-positive surface $\Sigma$. It is known that quasi-positive surfaces always minimize the Thurston norm; hence, since one has $\|L\|_{T}=\left\|L^{\prime}\right\|_{T}$ from the additivity of $\|\cdot\|_{T}$, we have that $\Sigma$ is the disjoint union of $\Sigma^{\prime}$ with some disks, where $\partial \Sigma^{\prime}=L^{\prime}$. This implies that $L^{\prime}$ is strongly quasi-positive.

At this point, in order to prove Theorem 3.1 we only need Lemma 3.3, which solves Exercise 8.4.9 (a) in [17.

Lemma 3.3. For every n-component link $L$ we have

$$
0 \leqslant \tau_{\max }(L)-\tau_{\min }(L) \leqslant n-1 .
$$

Proof. It follows from [5] because $\tau_{\min }(L)=-\tau_{\max }\left(L^{*}\right)$, for the symmetries of $c H F L^{-}(L)$, and $\tau_{\max }(L)$ is a slice-torus concordance invariant. To show this we can apply [5, Lemma 2.1]; then the only nontrivial fact to prove is that $\tau_{\max }$ coincides with $\tau$ for torus links, but this follows from [3, Theorem $1.4]$.

In particular, this result implies that $\tau(L)+\tau\left(L^{*}\right) \leqslant n-1$.

Proof of Theorem 3.1. From Lemma 3.2 we can suppose that $o(L)=0$. By using [3, Theorem 1.4] again, the fact that $L$ and $L^{*}$ are both strongly quasi-positive gives $2 \tau(L)-n=2 \tau\left(L^{*}\right)-n$, since $\|L\|_{T}=\left\|L^{*}\right\|_{T}$. Therefore, from Lemma 3.3 one has

$$
2 \tau(L) \leqslant n-1 \quad \text { and then } \quad\|L\|_{T} \leqslant-1,
$$

but this is impossible.

The following easier criterion can be used for alternating links. We recall that fibered links are always non-split and we denote by $\sigma(L)$ the signature and by $\nabla_{L}$ the Conway polynomial of $L$.

Proposition 3.4. A quasi-alternating n-component link is fibered and strongly quasi-positive if and only if $\nabla_{L}(z)=z^{-\sigma(L)}+f(z)$, where $f(z)$ has degree strictly smaller than $-\sigma(L)$.

Proof. It follows from Theorem 2.1 and the facts that $\widehat{H F L}(L)$ categorifies the polynomial $\left(t^{\frac{1}{2}}-t^{-\frac{1}{2}}\right)^{n-1} \cdot \nabla_{L}\left(t^{\frac{1}{2}}-t^{-\frac{1}{2}}\right)$ and quasi-alternating links are $\widehat{H F L}$-thin, see [17].

This result agrees with [1, where is shown that, for a non-split alternating link $L$, being strongly quasi-positive is equivalent to being positive and this happens if and only if $2 g_{3}(L)=1-n-\sigma(L)$. In fact, we also recall that for such a link $L$ one has $2 \tau(L)=n-1-\sigma(L)$. 


\begin{tabular}{|c|c|c|c|c|c|}
\hline name & strongly QP (SL) & name & strongly QP (SL) & name & strongly QP (SL) \\
\hline$\overline{\mathrm{L} 2 \mathrm{a} 1\{0\}}$ & $\begin{array}{l}\mathrm{Y}(0) \\
\end{array}$ & 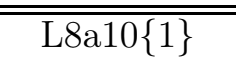 & " & 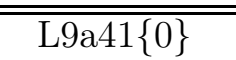 & $\begin{array}{l}\mathrm{N} \\
\end{array}$ \\
\hline $\operatorname{L} 2 \mathrm{a} 1\{1\}$ & $Y(0)$ & L8a14\{0\} & $\mathrm{Y}(6)$ & $\mathrm{L} 9 \mathrm{a} 42\{0\}$ & $\mathrm{N}$ \\
\hline $\mathrm{L} 4 \mathrm{a} 1\{1\}$ & $\mathrm{Y}(2)$ & $\mathrm{L} 8 \mathrm{a} 15\{0,0\}$ & $\mathrm{N}$ & $\operatorname{Laa} 42\{1\}$ & $\mathrm{N}$ \\
\hline $\mathrm{L} 5 \mathrm{a} 1\{0\}$ & $\mathrm{N}$ & L8a16\{0,0\} & $\mathrm{N}$ & $\mathrm{L} 9 \mathrm{a} 43\{1,0\}$ & $\mathrm{N}$ \\
\hline $\mathrm{L} 5 \mathrm{a} 1\{1\}$ & $\mathrm{N}$ & $\operatorname{La} 16\{1,1\}$ & $\mathrm{N}$ & $\operatorname{La} 943\{0,1\}$ & $\mathrm{N}$ \\
\hline $\mathrm{L} 6 \mathrm{a} 1\{0\}$ & $\mathrm{N}$ & $\mathrm{L} 8 \mathrm{a} 17\{1,1\}$ & $\mathrm{N}$ & $\operatorname{La} 43\{1,1\}$ & $\mathrm{N}$ \\
\hline $\mathrm{L} 6 \mathrm{a} 3\{0\}$ & $\mathrm{Y}(4)$ & L8a18 $\{0,0\}$ & $\mathrm{N}$ & $\mathrm{L} 9 \mathrm{a} 44\{0,0\}$ & $\mathrm{N}$ \\
\hline $\mathrm{L} 6 \mathrm{a} 4\{0,0\}$ & $\mathrm{N}$ & $\mathrm{L} 8 \mathrm{a} 18\{1,1\}$ & $\mathrm{N}$ & $\mathrm{L} 9 \mathrm{a} 44\{1,0\}$ & $\mathrm{N}$ \\
\hline $\mathrm{L} 6 \mathrm{a} 4\{1,0\}$ & $\mathrm{N}$ & $\mathrm{L} 8 \mathrm{a} 19\{0,0\}$ & $\mathrm{N}$ & $\mathrm{L} 9 \mathrm{a} 44\{0,1\}$ & $\mathrm{N}$ \\
\hline $\mathrm{L} 6 \mathrm{a} 4\{0,1\}$ & $\mathrm{N}$ & $\operatorname{L} 8 \mathrm{a} 19\{1,1\}$ & $\mathrm{N}$ & $\mathrm{L} 9 \mathrm{a} 46\{0,0\}$ & $\mathrm{N}$ \\
\hline $\mathrm{L} 6 \mathrm{a} 4\{1,1\}$ & $\mathrm{N}$ & $\mathrm{L} 8 \mathrm{a} 20\{0,0\}$ & $\mathrm{N}$ & L9a $46\{1,0\}$ & $\mathrm{N}$ \\
\hline $\operatorname{L} 6 a 5\{1,0\}$ & $\mathrm{N}$ & $\mathrm{L} 8 \mathrm{a} 20\{1,0\}$ & $\mathrm{N}$ & $\operatorname{La} 946\{0,1\}$ & $\mathrm{N}$ \\
\hline $\mathrm{L} 6 \mathrm{a} 5\{0,1\}$ & $\mathrm{N}$ & $\mathrm{L} 8 \mathrm{a} 21\{1,0,0\}$ & $\mathrm{N}$ & $\operatorname{La} 946\{1,1\}$ & $\mathrm{N}$ \\
\hline $\mathrm{L} 6 \mathrm{a} 5\{1,1\}$ & $\mathrm{N}$ & $\mathrm{L} 8 \mathrm{a} 21\{0,1,0\}$ & $\mathrm{N}$ & $\mathrm{L} 9 \mathrm{a} 47\{1,0\}$ & $\mathrm{N}$ \\
\hline $\mathrm{L} 6 \mathrm{n} 1\{0,0\}$ & $\mathrm{N}$ & $\operatorname{La} 21\{0,0,1\}$ & $\mathrm{N}$ & $\mathrm{L} 9 \mathrm{a} 47\{0,1\}$ & $\mathrm{N}$ \\
\hline $\operatorname{L} 6 n 1\{1,0\}$ & $\mathrm{N}$ & $\operatorname{L} 8 a 21\{1,0,1\}$ & $\mathrm{N}$ & $\mathrm{L} 9 \mathrm{a} 47\{1,1\}$ & $\mathrm{N}$ \\
\hline $\mathrm{L} 6 \mathrm{n} 1\{0,1\}$ & $\mathrm{Y}(3)$ & $\operatorname{L} 8 a 21\{0,1,1\}$ & $\mathrm{N}$ & L9a $48\{0,0\}$ & $\mathrm{N}$ \\
\hline $\mathrm{L} 6 \mathrm{n} 1\{1,1\}$ & $\mathrm{N}$ & $\mathrm{L} 8 \mathrm{a} 21\{1,1,1\}$ & $\mathrm{N}$ & L9a $49\{1,0\}$ & $\mathrm{N}$ \\
\hline L7a1 $\{0\}$ & $\mathrm{N}$ & L9a $2\{0\}$ & $\mathrm{N}$ & L9a $49\{0,1\}$ & $\mathrm{N}$ \\
\hline $\operatorname{L} 7 a 1\{1\}$ & $\mathrm{N}$ & L9a $2\{1\}$ & $\mathrm{N}$ & L9a $50\{0,0\}$ & $\mathrm{N}$ \\
\hline $\operatorname{L} 7 a 2\{1\}$ & $\mathrm{N}$ & L9a $5\{0\}$ & $\mathrm{N}$ & L9a $50\{1,0\}$ & $\mathrm{N}$ \\
\hline $\mathrm{L} 7 \mathrm{a} 3\{0\}$ & $\mathrm{N}$ & L9a6 $\{1\}$ & $\mathrm{N}$ & $\operatorname{La} 50\{1,1\}$ & $\mathrm{N}$ \\
\hline $\mathrm{L} 7 \mathrm{a} 3\{1\}$ & $\mathrm{N}$ & L9a $8\{0\}$ & $\mathrm{N}$ & L9a $51\{0,0\}$ & $\mathrm{N}$ \\
\hline $\mathrm{L} 7 \mathrm{a} 5\{1\}$ & $\mathrm{N}$ & L9a $8\{1\}$ & $\mathrm{N}$ & L9a51\{1,0\} & $\mathrm{N}$ \\
\hline $\mathrm{L} 7 \mathrm{a} 6\{0\}$ & $\mathrm{N}$ & L9a9 $\{0\}$ & $\mathrm{N}$ & $\operatorname{La} 51\{1,1\}$ & $\mathrm{N}$ \\
\hline $\mathrm{L} 7 \mathrm{a} 7\{0,0\}$ & $\mathrm{N}$ & L9a9 $\{1\}$ & $\mathrm{N}$ & L9a52\{1,0\} & $\mathrm{N}$ \\
\hline $\operatorname{L} 7 a 7\{1,0\}$ & $\mathrm{N}$ & L9a11 $\{0\}$ & $\mathrm{N}$ & L9a $52\{1,1\}$ & $\mathrm{N}$ \\
\hline $\operatorname{L} 7 a 7\{0,1\}$ & $\mathrm{N}$ & L9a12 $\{1\}$ & $\mathrm{N}$ & L9a $53\{0,0\}$ & $\mathrm{N}$ \\
\hline $\mathrm{L} 7 \mathrm{n} 1\{0\}$ & $\mathrm{Y}(4)$ & L9a14\{0\} & $\mathrm{N}$ & $\operatorname{La} 53\{1,0\}$ & $\mathrm{N}$ \\
\hline $\operatorname{Lnn} 1\{1\}$ & $\mathrm{N}$ & $\operatorname{Laa} 14\{1\}$ & $\mathrm{N}$ & $\operatorname{La} 953\{0,1\}$ & $\mathrm{N}$ \\
\hline $\operatorname{Ln} 2\{0\}$ & $\mathrm{N}$ & L9a16 $\{0\}$ & $\mathrm{N}$ & $\operatorname{La} 953\{1,1\}$ & $\mathrm{N}$ \\
\hline $\operatorname{Ln} 2\{1\}$ & $\mathrm{N}$ & L9a $20\{0\}$ & $\mathrm{N}$ & L9a $54\{0,0\}$ & $\mathrm{N}$ \\
\hline L8a1\{0\} & $\mathrm{N}$ & L9a $21\{0\}$ & $\mathrm{N}$ & $\mathrm{L} 9 \mathrm{a} 54\{1,0\}$ & $\mathrm{N}$ \\
\hline $\mathrm{L} 8 \mathrm{a} 1\{1\}$ & $\mathrm{N}$ & L9a22\{0\} & $\mathrm{N}$ & L9a $54\{0,1\}$ & $\mathrm{N}$ \\
\hline $\operatorname{Laa} 2\{0\}$ & $\mathrm{N}$ & $\operatorname{L} 9 a 24\{1\}$ & $\mathrm{N}$ & $\mathrm{L} 9 \mathrm{a} 54\{1,1\}$ & $\mathrm{N}$ \\
\hline $\operatorname{L} 8 \mathrm{a} 2\{1\}$ & $\mathrm{N}$ & $\operatorname{Laa} 26\{1\}$ & $\mathrm{N}$ & $\operatorname{Laa} 55\{0,0,0\}$ & $\mathrm{N}$ \\
\hline $\operatorname{L} 8 a 3\{1\}$ & $\mathrm{N}$ & L9a27\{0\} & $\mathrm{N}$ & L9a $55\{0,1,0\}$ & $\mathrm{N}$ \\
\hline $\mathrm{L} 8 \mathrm{a} 4\{0\}$ & $\mathrm{N}$ & L9a $28\{0\}$ & $\mathrm{N}$ & L9a $55\{0,0,1\}$ & $\mathrm{N}$ \\
\hline $\mathrm{L} 8 \mathrm{a} 4\{1\}$ & $\mathrm{N}$ & L9a29\{0\} & $\mathrm{N}$ & L9a $55\{1,0,1\}$ & $\mathrm{N}$ \\
\hline $\mathrm{L} 8 \mathrm{a} 5\{0\}$ & $\mathrm{N}$ & L9a31\{0\} & $\mathrm{N}$ & & \\
\hline $\operatorname{L} 8 \mathrm{a} 7\{1\}$ & $\mathrm{N}$ & L9a32\{1\} & $\mathrm{N}$ & & \\
\hline $\mathrm{L} 8 \mathrm{a} 8\{0\}$ & $\mathrm{N}$ & L9a33\{0\} & $\mathrm{N}$ & & \\
\hline $\mathrm{L} 8 \mathrm{a} 8\{1\}$ & $\mathrm{N}$ & L9a36 $\{0\}$ & $\mathrm{N}$ & & \\
\hline $\mathrm{L} 8 \mathrm{a} 9\{0\}$ & $\mathrm{N}$ & L9a38\{0\} & $\mathrm{N}$ & & \\
\hline $\operatorname{L} 8 a 9\{1\}$ & $\mathrm{N}$ & L9a39 $\{0\}$ & $\mathrm{N}$ & & \\
\hline
\end{tabular}

Table 1: Some fibered prime links with 9 or fewer crossings. In the second column we write whether or not the corresponding fibered link (or its mirror image) is strongly quasi-positive. In the latter case, the maximal self-linking number is denoted between brackets. 


\section{References}

[1] M. Boileau, S. Boyer and C. Gordon, Branched covers of quasipositive links and L-spaces, J. of Topology, 12 (2019), no. 2, pp. 536-576.

[2] A. Cavallo, The concordance invariant tau in link grid homology, Algebr. Geom. Topol., 18 (2018), no. 4, pp. 1917-1951.

[3] A. Cavallo, On Bennequin-type inequalities for links in tight contact 3-manifolds, J. Knot Theory Ramifications, 29 (2020), no. 8, 2050055.

[4] A. Cavallo, An invariant of Legendrian and transverse links from open book decompositions of contact 3-manifolds, Glasgow Math. J., 63 (2021), no. 2, pp. 451-483.

[5] A. Cavallo and C. Collari, Slice-torus concordance invariants and Whitehead doubles of links, Canad. J. Math., 72 (2020), no. 6, pp. 1423-1462.

[6] J. Etnyre and J. Van Horn-Morris, Fibered transverse knots and the Bennequin bound, Int. Math. Res. Not. IMRN, (2011), no. 7, pp. 1483-1509.

[7] P. Ghiggini, Knot Floer homology detects genus-one fibred knots, Amer. J. Math., 130 (2008), no. 5, pp. 1151-1169.

[8] E. Giroux, Géométrie de contact: de la dimension trois vers les dimensions supérieures, Proceedings of the International Congress of Mathematicians, Vol. II, Higher Ed. Press, Beijing, 2002, pp. 405414.

[9] J. Harer, How to construct all fibered knots and links, Topology, 21 (1982), no. 3, pp. 263-280.

[10] K. Hayden, Minimal braid representatives of quasipositive links, Pacific J. Math., 295 (2018), no. 2, pp. 421-427.

[11] M. Hedden, Notions of positivity and the Ozsváth-Szabó concordance invariant, J. Knot Theory Ramifications, 19 (2010), no. 5, pp. 617-629.

[12] K. Honda, W. Kazez and G. Matić, On the contact class in Heegaard Floer homology, J. Differential Geom., 83 (2009), no. 2, pp. 289-311.

[13] A. Kawauchi, A survey of knot theory, Birkhäuser Verlag, Basel, 1996, pp. xxii and 420.

[14] C. Livingston and A. H. Moore, KnotInfo: Table of Link Invariants, http://www.indiana.edu/ knotinfo, December 27, 2019.

[15] Y. Ni, Knot Floer homology detects fibred knots, Invent. Math., 170 (2007), no. 3, pp. 577-608.

[16] Y. Ni, Link Floer homology detects the Thurston norm, Geom. Topol., 13 (2009), no. 5, pp. 29913019.

[17] P. Ozsváth, A. Stipsicz and Z. Szabó, Grid homology for knots and links, AMS, volume 208 of Mathematical Surveys and Monographs, 2015.

[18] P. Ozsváth and Z. Szabó, Holomorphic disks and topological invariants of closed three-manifolds, Ann. of Math. (2), 159 (2004), no. 3, pp. 1159-1245.

[19] P. Ozsváth and Z. Szabó, Heegaard Floer homology and contact structures, Duke Math. J., 129 (2005), no. 1, pp. 39-61. 
[20] P. Ozsváth and Z. Szabó, Heegaard diagrams and Floer homology, International Congress of Mathematicians Vol. II, pp. 1083-1099, Eur. Math. Soc., Zürich, 2006.

[21] P. Ozsváth and Z. Szabó, Holomorphic disks, link invariants and the multi-variable Alexander polynomial, Algebr. Geom. Topol., 8 (2008), no. 2, pp. 615-692.

[22] J. Stallings, Constructions of fibred knots and links, Algebraic and geometric topology, Part 2, pp. 55-60, Proc. Sympos. Pure Math., XXXII, Amer. Math. Soc., Providence, R.I., 1978.

[23] W. Thurston, A norm for the homology of 3-manifolds, Mem. Amer. Math. Soc., 59 (1986), no. 339, pp. i-vi and 99-130. 